Sādhanā Vol. 40, Part 6, September 2015, pp. 1913-1924. (C) Indian Academy of Sciences

\title{
On Reynolds stress and neutral azimuthal modes in the stability problem of swirling flows with radius-dependent density
}

\author{
H DATTU* and M SUBBIAH
}

Department of Mathematics, Pondicherry University, Pondicherry 605014, India

e-mail:dattuhalle@gmail.com; malaisubbiah@gmail.com

MS received 24 December 2013; revised 3 March 2015; accepted 7 April 2015

\begin{abstract}
We consider the linear stability problem of inviscid, incompressible swirling flows with radius-dependent density with respect to two-dimensional disturbances. Some results of Miles on the parallel flow stability theory are extended to the swirling flow stability theory. In particular, series solutions for the stability equation for swirling flows are obtained and these solutions are used in the study of the variation of the Reynolds stress. For singular neutral modes it is shown that the Reynolds stress varies like the inverse square of the radial distance in agreement with the homogeneous flow result of Maslowe \& Nigam. It is also proved that singular neutral modes do not exist whenever the value of the Richardson number at the critical layer exceeds one quarter.
\end{abstract}

Keywords. Hydrodynamic stability; swirling flows; inviscid flows; variable density; Reynolds stress.

\section{Introduction}

The stability of swirling flows has been studied extensively and for the vast literature on this problem one may be referred to the books by Chandrasekhar (1961), Drazin \& Reid (1981), and Chossat \& Iooss (1994). For analytical studies on this problem one considers a basic flow with azimuthal and axial velocity components and general three-dimensional disturbances (see, for example, Howard \& Gupta (1962)). However the stability of basic flows with only an azimuthal velocity component to infinitesimal azimuthal disturbances has also been studied in many works (see, for example, Drazin \& Reid (1981)). In cylindrical polar coordinates $(r . \theta, z)$ the basic flow velocity is given by $(0, V(r), 0)$ and the basic flow density is $\rho_{0}(r)$ while the basic flow pressure $P_{0}(r)$ is calculated from the Euler equations. The angular velocity $\Omega(r)=\frac{V(r)}{r}$. The flow domain is the annular region between two infinite concentric cylinders with radii $R_{1}$ and $R_{2}$ where $0<R_{1}<R_{2}<\infty$. Here the cylinders can rotate and create a swirling flow by the influence of viscosity and a density variation can be created by maintaining the two cylinders

${ }^{*}$ For correspondence 
at different temperatures. But, as a first step in the stability analysis, we consider the inviscid stability analysis only ignoring the viscous and diffusive effects.

If the disturbed flow is given by $(u, V+v, 0)$ and the disturbances are azimuthal disturbances, that is, disturbances are of the form (function of $r) e^{i m(\theta-c t)}$ then $m$ is an integer and it is called the azimuthal wave number and $c=c_{r}+i c_{i}$ is called the (complex) phase velocity. The boundary conditions satisfied by the disturbances is $\hat{u}=0$ at $r=R_{1}, R_{2}$ where $u=\hat{u}(r) e^{i m(\theta-c t)}$ is the axial disturbance velocity. Fung \& Kurzweg (1975) have found the linear stability equation for this problem and studied the instability of some specific basic flows. Then Fung (1983) obtained some general analytical results for this problem, in particular he obtained the semicircular and semielliptical instability regions for basic flows satisfying the condition $a b\left(D \rho_{0}\right) \geq 0$ where $a=\min \Omega(r), b=\max \Omega(r)$, and the differential operator $D$ is defined by $D=\frac{d}{d r}$ and the $\min$ and $\max$ stand for minimum and maximum taken over $\left[R_{1}, R_{2}\right]$. Moreover, Fung (1983) has defined the Richardson number $J$ by $J=\frac{\Omega^{2}\left(D \rho_{0}\right)}{\rho_{0} r(D \Omega)^{2}}$ and has found that a necessary condition for instability is that the minimum of the Richardson number is less than one quarter.

Recently this problem has been studied in Dattu \& Subbiah (2014a) where an improved instability region given by a generalized semiellipse theorem has been found for arbitrary angular velocity profiles. This improved instability region has also been used to find an estimate for the growth rate of unstable disturbances. The two-dimensional instability of the Rankine vortex with variable density has been studied recently in Dixit \& Govindarajan (2011). Since $D \Omega=0$ in this case they have studied the instability with respect to the Atwood number rather than the Richardson number. Subsequently Dixit \& Govindarajan (2011) have considered the stability of smoothly varying angular velocity profiles for which $D \Omega$ is not zero but the growth rate variations with respect to the Atwood number alone are presented and the Richardson number is not involved in their analysis. Also the instability of inviscid incompressible swirling flows with variable density with respect to two-dimensional disturbances has been studied asymptotically and numerically in Di Pierro \& Abid (2010). For slowly varying velocity profiles Di Pierro \& Abid (2010) have studied the growth rate of an unstable disturbance in the limit $|m|>>1$ and found that their asymptotic result agrees with their numerical result. Subsequently a numerical study of the nonlinear stability of swirling flows with variable density was made in Di Pierro \& Abid (2012) and it was found that the linear and asymptotic results of their earlier paper are in agreement with their new results. In addition to their numerical results a brief discussion of the importance of the swirling flows and their stability is also given in their paper. It is stated that swirling flows are important for many application devices and as a fundamental problem considering their relevance to aircraft trailing vortices, vortical transport of momentum and energy in meteorology, and vortex breakdown. Hence they are widely studied. However, the physical mechanism of their instabilities, when density variations are present, are not generally discussed. Moreover, Di Pierro \& Abid (2012) have pointed out that, in a variable density swirling flow there are ingredients for the development of two fundamental instabilities: the Rayleigh-Taylor instability due to density variations and Taylor-Couette instability due to differential rotation. Furthermore it is pointed out that the Rayleigh-Taylor instability is characterized by "bubbles" for positively buoyant fluids and "spikes" for negatively buoyant fluids. It may be noted here that the instability of swirling flows of homogeneous fluid has been studied in Le Dizès (2000) with respect to azimuthal disturbances where a nonlinear critical layer analysis has been developed. Moreover, in the recent work of Maslowe \& Nigam (2008) linear and nonlinear stability of homogeneous swirling flows with respect to general three dimensional disturbances has been studied. In the linear analysis they have studied the radial variation of the Reynolds stress and found that the Reynolds stress for neutral modes is proportional to 
$r^{-2}$ which is different from that of parallel shear flows in which case the Reynolds stress is a constant. Also it is remarked in Maslowe \& Nigam (2008) that smoothly varying angular velocity profiles are more realistic in some contexts and this fact has also been mentioned in Spalart (1998).

In the present paper we consider the stability problem of swirling flows with variable density of inviscid incompressible fluids confined between two infinite concentric cylinders at $r=R_{1}$ and $r=R_{2}$ where $0<R_{1}<R_{2}<\infty$ with respect to azimuthal disturbances. As stated earlier some analytical results on this problem were obtained in Fung (1983). These results of Fung (1983) were obtained by extending the corresponding results of parallel flow theory to swirling flow context. As is well known the Taylor-Goldstein problem of hydrodynamic stability deals with stability of density stratified shear flows of incompressible, inviscid fluid (see, for example, Drazin \& Reid (1981)). A seminal work on this problem is Miles (1961). In this paper Miles proved that a necessary condition for instability is that the (local) Richardson number must be less than one quarter somewhere in the flow domain. However the proof of this result was restricted to velocity and density profiles that are analytic functions of the vertical coordinate variable. Howard (1961) gave a proof of this result without these restrictions, and this result is known as the Miles-Howard theorem. Another interesting result of Howard (1961) is his semicircle theorem according to which the complex phase velocity of unstable modes should lie inside a semicircle in the upper half-plane. Noticing that Howard's semicircle does not depend on the stratification parameter Kochar \& Jain (1979) proved their semi-ellipse theorem giving an instability region that depends the minimum Richardson number and lying inside Howard's semi-circle. Fung's results mentioned earlier are extensions of these results to swirling flows. However Miles (1961) contains many more interesting results that have not been extended to swirling flows. In particular Miles (1961) classified the neutral modes into two classes, namely, the neutral modes that are contiguous to unstable modes and consequently lie on the stability boundary and the isolated modes. As he found that only singular neutral modes can lie on the stability boundary these modes were studied by him in detail. Another aspect of the problem studied by him is the Reynolds stress that transfers energy from the basic flow to the disturbance. As the Taylor-Goldstein equation is a second order ordinary differential equation Miles (1961) found two linearly independent solutions around the critical layer at which the phase velocity of wave equals the basic flow velocity. These solutions were subsequently used by him to study the Reynolds stress and its variation. In particular it is seen that the Reynolds stress is a constant for neutral modes when the basic parallel flow is monotonic. An important result of Miles (1961) is that singular neutral modes do not exist if the value of the Richardson number at the critical layer exceeds one quarter. It may be remarked here that these results of Miles (1961) play important roles in the linear viscous critical layer theory (see, for example, Maslowe \& Spiteri (2013)) and in the nonlinear critical layer theory (see, for example, Kelly \& Maslowe 1970)). In particular Maslowe \& Spiteri (2013) have found that a neutral mode can change the velocity profile of the basic flow and consequently its stability properties and that this conclusion is obtained by studying the variation of the Reynolds stress in the critical layer.

Now we state the problem that is being studied in the present paper. The main aspect of the problem discussed in this paper is the variation of Reynolds stress and the existence of neutral modes. As mentioned earlier this aspect has been discussed in Maslowe \& Nigam (2008) for the special case of the stability of homogeneous flows but with the general case of three dimensional disturbances. But the Richardson number defined in their paper depends on the axial wave number and not just on the basic flow variables. In our paper we consider the more general case of density stratified swirling flows with two-dimensional disturbances. The Richardson number 
here is $J=\frac{\Omega^{2}\left(D \rho_{0}\right)}{\rho_{0} r(D \Omega)^{2}}$ as defined in Fung (1983) and it depends only on the basic flow variables. In the stability analysis of density stratified parallel shear flows of inviscid incompressible fluids Miles (1961) has shown that the Reynolds stress is a constant for neutral modes. In the present paper we show that the Reynolds stress for neutral azimuthal modes varies like $r^{-2}$ for variable density fluids also. The point $r=r_{c}$ where $\Omega-c=0$ is called a critical layer that is the layer in flow region where the phase velocity of the neutral azimuthal mode equals the basic angular velocity. And it is shown here that the algebraic sum of the jumps in the Reynolds stress across critical layers is zero. Consequently there is no jump in the Reynolds stress across the critical layer for basic swirling flows with monotone angular velocity profiles. Moreover we find the Frobenius series solution of the stability equation and also use them to show that singular neutral modes do not exist when $J_{c}>\frac{1}{4}$ where $J_{c}=J\left(r_{c}\right)$ is the value of the Richardson number at a critical layer. This result is analogous to the corresponding result of Miles (1961) on the stability problem of density stratified parallel shear flows.

It may be observed here that the results of the present study; except for the one on the radial variation of the Reynolds stress for neutral modes, largely resemble those of Miles (1961). However, the current study and the one by Miles (1961) are for different flow configurations; swirling and parallel shear flows, respectively. The reason for this resemblance of the results is the analogy between the centrifugal acceleration $\frac{V^{2}}{r}$ and the gravitational acceleration, in density stratified fluids. This analogy was originally observed by Rayleigh (cf. Howard \& Gupta 1962) and it has been exploited by Howard \& Gupta (1962) in the stability analysis of swirling flows, when an axial velocity is also present, with respect to axisymmetric disturbances. When one considers only the azimuthal disturbances, as done in the present paper, the axial velocity does not play any role (see Howard \& Gupta 1962) and one has the stability equation of Fung \& Kurzweg (1975).

\section{Eigenvalue problem}

The linear stability problem of inviscid incompressible but density stratified swirling flows between two infinite concentric co-axial cylinders at $r=R_{1}, R_{2}$ where $0<R_{1}<R_{2}<\infty$ with respect to azimuthal disturbances of the form (function of $r$ ) $e^{i m(\theta-c t)}$ is given by an eigen value problem consisting of a second order ordinary differential equation of Fung \& Kurzweg (1975). The Fung \& Kurzweg (1975) equation is given in terms of the unknown $\hat{u}(r)$ where $\hat{u}(r) e^{i m(\theta-c t)}$ is the axial disturbance velocity. If $\phi(r) e^{i m(\theta-c t)}$ is the disturbance streamfunction then it is related to the disturbance axial velocity by the relation $\phi(r)=\frac{i r \hat{u}(r)}{m}$ and the Fung \& Kurzweg (1975) equation gives stability equation

$$
\rho_{0}\left(D_{*} D-\frac{m^{2}}{r^{2}}\right) \phi+\left(D \rho_{0}\right)(D \phi)+\left\{\frac{\Omega^{2}\left(D \rho_{0}\right)}{r(\Omega-c)^{2}}-\frac{D\left(\rho_{0} Z\right)}{r(\Omega-c)}\right\} \phi=0,
$$

where $Z=r D \Omega+2 \Omega$ is the basic vorticity, the operator $D_{*}$ is defined to be $D_{*}=D+\frac{1}{r}$, and the boundary conditions are

$$
\phi=0 \quad \text { at } r=R_{1}, R_{2} \text {. }
$$

In this equation the azimuthal wave number ' $m$ ' appears as $m^{2}$ and so we can take $m>0$ without loss of generality. 


\section{Reynolds stress and neutral modes}

Here we are considering, as stated in the introduction, the motion of an inviscid incompressible but density stratified fluid between concentric cylinders of radii $R_{1}$ and $R_{2}$ with $0<R_{1}<R_{2}<\infty$. The basic flow has velocity $(0, V(r), 0)$, pressure $P_{0}(r)$ and density $\rho_{0}(r)$, where the pressure is related to the velocity and density through the relation $\frac{d P_{0}}{d r}=\rho_{0}\left(\frac{V^{2}}{r}\right)$. We consider the disturbed flows to be given by the velocity $(u, V+v, 0)$, pressure $P_{0}(r)+p$, and density $\rho_{0}(r)+\rho$. If the disturbances are infinitesimal and azimuthal then the disturbance variables satisfy the following equations:

$r$-momentum equation

$$
\rho_{0}\left(\frac{\partial u}{\partial t}+\frac{V}{r} \frac{\partial u}{\partial \theta}-\frac{2 V}{r} v\right)-\frac{V^{2}}{r} \rho=-\frac{\partial p}{\partial r},
$$

$\theta$-momentum equation

$$
\rho_{0}\left(\frac{\partial v}{\partial t}+u \frac{d V}{d r}+\frac{V}{r} \frac{\partial v}{\partial \theta}+\frac{V}{r} u\right)=-\frac{1}{r} \frac{\partial p}{\partial \theta},
$$

incompressible equation

$$
\frac{\partial u}{\partial r}+\frac{u}{r}+\frac{1}{r} \frac{\partial v}{\partial \theta}=0
$$

continuity equation

$$
\frac{\partial \rho}{\partial t}+u \frac{d \rho_{0}}{d r}+\frac{V}{r} \frac{\partial \rho}{\partial \theta}=0
$$

A fluid flow is incompressible when the volume of any moving portion of fluid is constant and this is equivalent to the condition of zero divergence of the fluid velocity ; (3.3) follows from this. The equation of continuity follows from the conservation of mass principle, by which the mass of any moving portion of fluid is constant; for an incompressible flow this reduces to the fact that the density of a fluid particle in motion is a constant, and (3.4) follows.

Multiply Eq. (3.1) by $r u$, multiply Eq. (3.2) by $r v$, add them and then integrate the resultant equation to get the equation,

$$
\begin{array}{r}
\iint \rho_{0}\left[\frac{\partial}{\partial t}+\Omega \frac{\partial}{\partial \theta}\right]\left(\frac{u^{2}+v^{2}}{2}\right) r d r d \theta-\iint \rho r \Omega^{2} u r d r d \theta \\
+\iint \rho_{0} r D \Omega u v r d r d \theta=-\iint\left(r \frac{\partial(u p)}{\partial r}-r p \frac{\partial u}{\partial r}+\frac{\partial(v p)}{\partial \theta}-p \frac{\partial v}{\partial \theta}\right) d r d \theta .
\end{array}
$$

The second term in the first integral is

$$
\begin{aligned}
\iint \rho_{0} \Omega \frac{\partial}{\partial \theta}\left(\frac{u^{2}+v^{2}}{2}\right) r d r d \theta & =\int \rho_{0} \Omega\left(\int \frac{\partial}{\partial \theta} \frac{u^{2}+v^{2}}{2} d \theta\right) r d r \\
& =\int \rho_{0} \Omega\left(\frac{u^{2}+v^{2}}{2}\right)_{\theta_{0}}^{\theta_{0}+\frac{2 \pi}{m}} r d r \\
& =0 \text { (by periodicity in } \theta \text { ). }
\end{aligned}
$$


Thus Eq. (3.5) becomes

$$
\begin{array}{r}
\iint \rho_{0}\left[\frac{\partial}{\partial t}\left(\frac{u^{2}+v^{2}}{2}\right)\right] r d r d \theta-\iint \rho r \Omega^{2} u r d r d \theta+\iint \rho_{0} r D \Omega u v r d r d \theta \\
=-\iint\left(r \frac{\partial(u p)}{\partial r}+\frac{\partial}{\partial \theta}(v p)\right) d r d \theta+\iint p r\left(\frac{\partial u}{\partial r}+\frac{1}{r} \frac{\partial v}{\partial \theta}\right) d r d \theta
\end{array}
$$

But from Eq. (3.3) we have $\frac{\partial u}{\partial r}+\frac{1}{r} \frac{\partial v}{\partial \theta}=-\frac{u}{r}$, and use of this in the above equation gives

$$
\begin{gathered}
\iint \rho_{0}\left[\frac{\partial}{\partial t}\left(\frac{u^{2}+v^{2}}{2}\right)\right] r d r d \theta-\iint \rho r \Omega^{2} u r d r d \theta+\iint \rho_{0} r D \Omega u v r d r d \theta \\
\left.=-\iint\left(r \frac{\partial(u p)}{\partial r}+u p\right) d r d \theta-\iint \frac{\partial}{\partial \theta}(v p)\right) d r d \theta .
\end{gathered}
$$

Now using boundary conditions and periodicity in $\theta$ we see that the right-hand side vanishes and so we have

$$
\iint \rho_{0}\left[\frac{\partial}{\partial t}\left(\frac{u^{2}+v^{2}}{2}\right)\right] r d r d \theta-\iint \rho r \Omega^{2} u r d r d \theta+\iint \rho_{0} r D \Omega u v r d r d \theta=0 .
$$

Since $\frac{D \rho}{D t}=0$ the density of fluid layer at $r$ at present is equal to the basic flow density at $r-\eta$ where $\eta$ is the displacement of a fluid layer in the radial direction; that is,

$$
\begin{aligned}
\rho_{0}(r)+\rho(r, \theta, t) & =\rho_{0}(r-\eta) \\
& =\rho_{0}(r)+\left(D \rho_{0}\right)(r)(-\eta)+\ldots . .
\end{aligned}
$$

Under linearization this gives the following relation between the perturbation density and the displacement of the fluid layer:

$$
\rho=-\left(D \rho_{0}\right) \eta
$$

Moreover, the radial component of the disturbance velocity is given by

$$
u=\left(\frac{\partial}{\partial t}+\Omega \frac{\partial}{\partial \theta}\right) \eta
$$

Using (3.10) and (3.11) in (3.8) we have the following equation:

$$
\begin{aligned}
\frac{d}{d t} \iint \rho_{0}\left[\left(\frac{u^{2}+v^{2}}{2}\right)+r \Omega^{2} \frac{\left(D \rho_{0}\right)}{\rho_{0}} \frac{\eta^{2}}{2}\right] r d r d \theta & =-\iint \rho_{0}(D \Omega) u v r^{2} d r d \theta \\
& =\int \tau(D \Omega) r^{2} d r
\end{aligned}
$$

where,

$$
\tau=-\frac{m}{2 \pi} \int_{\theta_{0}}^{\theta_{0}+\frac{2 \pi}{m}} \rho_{0} u v d \theta
$$

is the Reynolds stress. 
Thus it is seen that the transfer of energy from the mean flow to a growing disturbance is done by the Reynolds stress.

Now we shall discuss the solution of the stability equation (2.1).

Equation (2.1) can be rewritten as

$$
\rho_{0}\left(D^{2} \phi+\frac{D \phi}{r}-\frac{m^{2} \phi}{r^{2}}\right)+\left(D \rho_{0}\right)(D \phi)+\left\{\frac{\Omega^{2}\left(D \rho_{0}\right)}{r(\Omega-c)^{2}}-\frac{D\left(\rho_{0} Z\right)}{r(\Omega-c)}\right\} \phi=0,
$$

Use the transformation $\phi=\frac{X}{\sqrt{r \rho_{0}}}$ to get the equation in the normal form; that is,

$$
D^{2} X+\left\{\frac{1-4 m^{2}}{4 r^{2}}+\frac{\left(D \rho_{0}\right)^{2}}{4 \rho_{0}^{2}}-\frac{D \rho_{0}}{2 r \rho_{0}}-\frac{D^{2} \rho_{0}}{2 \rho_{0}}+\frac{\Omega^{2}\left(D \rho_{0}\right)}{\rho_{0} r(\Omega-c)^{2}}-\frac{D\left(\rho_{0} Z\right)}{r \rho_{0}(\Omega-c)}\right\} X=0
$$

Let $r=r_{c}$ be a point where $\Omega=c$. Then Frobenius series solutions of Eq. (3.15) are of the form

$$
X_{ \pm}(r)=\left(r-r_{c}\right)^{\frac{1 \pm v}{2}} w_{ \pm}(r)
$$

where $v=\sqrt{1-4 J_{c}}, J_{c}=\left(\frac{\Omega^{2}\left(D \rho_{0}\right)}{\rho_{0} r(D \Omega)^{2}}\right)_{r=r_{c}}$ and the analytical functions $w_{ \pm}(r)$ are given by

$$
w_{ \pm}(r)=1+\left\{J \frac{\left(D^{2} \Omega\right)}{(D \Omega)}-\frac{2 J D \Omega}{\Omega}-J \frac{\left(D^{2} \rho_{0}\right)}{D \rho_{0}}+\frac{J}{r}+\frac{D\left(\rho_{0} Z\right)}{(D \Omega) \rho_{0} r}\right\}_{r_{c}} \frac{r-r_{c}}{1 \pm v}+\ldots .
$$

where $(\Omega-c)=(c-\Omega) e^{\mp i \pi},(D \Omega)_{c} \gtrless 0$ are two branches and $w_{+}^{*}=w_{-}^{*}$ stands for complex conjugation. Actually we take $r-r_{c}=\left|r-r_{c}\right|$ when $r>r_{c}$ and $r-r_{c}=-\left|r-r_{c}\right|$ when $r<r_{c}$. Here we consider neutral modes that are limits of unstable modes and for unstable modes $c_{i}>0$. So we have to take $-1=e^{\mp i \pi}$ according as $(D \Omega)_{c} \gtrless 0$.

The Wronskian of the solutions (3.16) at $r=r_{c}$ is given by

$$
W\left\{X_{+}, X_{-}\right\}=X_{+} D X_{-}-X_{-} D X_{+}=-v
$$

and it is seen that the two solutions are linearly independent if $v \neq 0$; that is when $J_{c} \neq 1 / 4$. Also the conjugate functions are given by

$$
\left.\begin{array}{ll}
X_{ \pm}^{*}(r)=X_{ \pm}(r) e^{i \pi(1 \pm v) S\left(r-r_{c}\right)}, & J_{c}<\frac{1}{4} \\
X_{ \pm}^{*}(r)=X_{\mp}(r) e^{\pi(i \pm \mu) S\left(r-r_{c}\right)}, & J_{c}>\frac{1}{4}
\end{array}\right\}
$$

where $\mu=-i v$ and the function $S\left(r-r_{c}\right)$ is defined to be

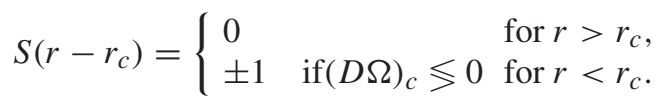

Since the perturbation streamfunction is $\phi(r) e^{i m(\theta-c t)}$ the disturbance velocity components are given by

$$
\left.\begin{array}{r}
u=\frac{i m \phi}{r} e^{i m(\theta-c t)}=i m r^{\frac{-3}{2}} \rho_{0}^{\frac{-1}{2}} X e^{i m(\theta-c t)}, \\
v=-(D \phi) e^{i m(\theta-c t)}=-\left[D\left(r^{\frac{-1}{2}} \rho_{0}^{\frac{-1}{2}}\right) X+r^{\frac{-1}{2}} \rho_{0}^{\frac{-1}{2}}(D X)\right] e^{i m(\theta-c t)} .
\end{array}\right\} .
$$


Noting that the velocity variables are given by the real part of those given in (3.21), we see that the Reynolds stress is given by

$$
\begin{aligned}
\tau & =\frac{-i m}{4 r^{2}}\left(X\left(D X^{*}\right)-X^{*}(D X)\right) e^{2 m c_{i} t}, \\
& =\frac{m}{r^{2}}\left(X^{*}(D X)\right)_{i} e^{2 m c_{i} t},
\end{aligned}
$$

where $(.)_{i}$ denotes imaginary part of the quantity inside the bracket.

It follows that

$$
\begin{aligned}
\frac{\partial}{\partial r}\left(r^{2} \tau\right) & =\frac{i m}{4}\left(X\left(D^{2} X^{*}\right)-X^{*}\left(D^{2} X\right)\right) e^{2 m c_{i} t}, \\
\text { i.e } \frac{\partial}{\partial r}\left(r^{2} \tau\right) & =\frac{m}{2}\left(X^{*}\left(D^{2} X\right)\right)_{i} e^{2 m c_{i} t} .
\end{aligned}
$$

But from Eq. (3.15) we have

$$
D^{2} X=-\left\{\frac{1-4 m^{2}}{4 r^{2}}+\frac{\left(D \rho_{0}\right)^{2}}{4 \rho_{0}^{2}}-\frac{D \rho_{0}}{2 r \rho_{0}}-\frac{D^{2} \rho_{0}}{2 \rho_{0}}+\frac{\Omega^{2}\left(D \rho_{0}\right)}{\rho_{0} r(\Omega-c)^{2}}-\frac{D\left(\rho_{0} Z\right)}{r \rho_{0}(\Omega-c)}\right\} X,
$$

and taking the complex conjugate of the above equation gives

$$
\left(D^{2} X^{*}\right)=-\left\{\frac{1-4 m^{2}}{4 r^{2}}+\frac{\left(D \rho_{0}\right)^{2}}{4 \rho_{0}^{2}}-\frac{D \rho_{0}}{2 r \rho_{0}}-\frac{D^{2} \rho_{0}}{2 \rho_{0}}+\frac{\Omega^{2}\left(D \rho_{0}\right)}{\rho_{0} r\left(\Omega-c^{*}\right)^{2}}-\frac{D\left(\rho_{0} Z\right)}{r \rho_{0}\left(\Omega-c^{*}\right)}\right\} X^{*} .
$$

Using (3.25)and (3.26) in Eq. (3.24) we have

$$
\frac{\partial}{\partial r}\left(r^{2} \tau\right)=\frac{m c_{i}}{2 \rho_{0}}\left\{\frac{D\left(\rho_{0} Z\right)}{|\Omega-c|^{2}}-\frac{2\left(D \rho_{0}\right) \Omega^{2}\left(\Omega-c_{r}\right)}{|\Omega-c|^{4}}\right\}|X|^{2} e^{2 m c_{i} t} .
$$

For neutral modes it follows that the right-hand side of (3.27) becomes zero and we have $\tau=\frac{\tau_{0}}{r^{2}}$ where $\tau_{0}$ is a constant. This $r^{-2}$ behavior of $\tau$ contrasts with the case of parallel shear flow, where $\tau=$ constant (cf. Drazin \& Reid 1981; Miles 1961).

Remark 3.1. For homogeneous fluids for which $\rho_{0}=$ constant this behavior of the Reynolds stress has been obtained in Maslowe \& Nigam (2008).

For monotonic angular velocity profiles $\Omega=c$ only at one point and since

$$
\tau\left(R_{1}\right)=0=\tau\left(R_{2}\right)
$$

we have the following theorem.

Theorem 3.2. For monotonic angular velocity profiles $\Omega(r)$ the Reynolds stress for any neutral mode vanishes identically in the flow domain.

If the angular velocity is not monotonic then it is possible that $\Omega=c$ at more than one value of $r$ and in this case $\tau$ can become discontinuous at these layers. 
Now we pose general solution to Eq. (3.15) in the form

$$
X=A X_{+}+B X_{-},
$$

where $A$ and $B$ are constants, and for neutral modes substituting this in (3.22) we obtain

$$
\tau=\frac{m}{2 r^{2}}\left(|A|^{2} D X_{+} X_{+}^{*}+|B|^{2} D X_{-} X_{-}^{*}+A B^{*} D X_{+} X_{-}^{*}+A^{*} B X_{+}^{*} D X_{-}\right)_{i} .
$$

If $J_{c}<\frac{1}{4}$, then

$$
\begin{aligned}
\left(D X_{+}\right) X_{-}^{*} & =\left(\left(D X_{+}\right) X_{-}^{*}\right) e^{i \pi(1+v) S} \\
& =\left(v+X_{+}\left(D X_{-}\right)\right)^{*} e^{-i \pi(1+v) S}(\text { from }(3.18)) \\
& =v e^{-i \pi(1+v) S}+X_{+}\left(D X_{-}^{*}\right) .
\end{aligned}
$$

Using this along with the first equation of (3.19), Eq. (3.30) reduces to

$$
\tau=\frac{m v}{2 r^{2}}\left\{A B^{*} e^{-i \pi(1+v) S}\right\}_{i} ; \quad\left(J_{c}<\frac{1}{4}\right) .
$$

Similarly we can obtain for $J_{c}>\frac{1}{4}$ the following relation:

$$
\tau=\frac{m \mu}{4 r^{2}}\left\{|A|^{2} e^{\pi(i+\mu) S}-|B|^{2} e^{\pi(i-\mu) S}\right\} ; \quad\left(J_{c}>\frac{1}{4}\right) .
$$

Using the boundary conditions (3.28) on (3.32) we have either $A=0$ or $B=0$ and hence we have the following theorem.

Theorem 3.3. For a singular neutral mode $X$ must be simply proportional to either $X_{+}$or $X_{-}$ when $0<J_{c}<\frac{1}{4}$.

Similarly from (3.33) we have the following theorem.

Theorem 3.4. Singular neutral modes cannot exist for monotonic $\Omega(r)$ if $J_{c}>\frac{1}{4}$ in $\left[R_{1}, R_{2}\right]$.

The above results on the existence of singular neutral modes are the swirling flow extensions of the corresponding results of Miles (1961). It has been shown in Dattu \& Subbiah (2014b) that the series solutions presented above play important roles in the nonlinear critical layer analysis of swirling flows with variable density. However the above results on the Reynolds stress are similar to the corresponding results of Maslowe \& Nigam (2008). The main difference between our results and those of Maslowe \& Nigam (2008) is that our results are for basic flows with variable density while those of Maslowe \& Nigam (2008) are for constant density flows. Consequently the Richardson number considered in our paper is very different from that considered in Maslowe $\&$ Nigam (2008). Now we shall derive a formula for the jump in the Reynolds stress across a critical layer which has not got a corresponding result in Maslowe \& Nigam (2008).

Integrating Eq. (3.27) from $r_{c}-\epsilon$ to $r_{c}+\epsilon$ we have

$$
\left(r^{2} \tau\right)_{r_{c}-\epsilon}^{r_{c}+\epsilon}=\int_{r_{c}-\epsilon}^{r_{c}+\epsilon} \frac{m c_{i}}{2 \rho_{0}}\left\{\frac{D\left(\rho_{0} Z\right)}{|\Omega-c|^{2}}-\frac{2\left(D \rho_{0}\right) \Omega^{2}\left(\Omega-c_{r}\right)}{|\Omega-c|^{4}}\right\}|X|^{2} e^{2 m c_{i} t} d r .
$$


Consequently the jump in $r^{2} \tau$ denoted by $\left[r^{2} \tau\right]_{r_{c}}$ is given by

$$
\begin{aligned}
{\left[r^{2} \tau\right]_{r_{c}}=} & \lim _{\epsilon \rightarrow 0} \lim _{c_{i} \rightarrow 0^{+}} \int_{r_{c}-\epsilon}^{r_{c}+\epsilon} \frac{m c_{i}}{2 \rho_{0}}\left\{\frac{D\left(\rho_{0} Z\right)}{|\Omega-c|^{2}}-\frac{2\left(D \rho_{0}\right) \Omega^{2}\left(\Omega-c_{r}\right)}{|\Omega-c|^{4}}\right\}|X|^{2} e^{2 m c_{i} t} d r \\
= & \lim _{\epsilon \rightarrow 0} \lim _{c_{i} \rightarrow 0^{+}} \frac{m e^{2 m c_{i} t}}{2} \int_{r_{c}-\epsilon}^{r_{c}+\epsilon}\left\{\frac{D\left(\rho_{0} Z\right)}{\rho_{0}}-\frac{2\left(D \rho_{0}\right) \Omega^{2}\left(\Omega-c_{r}\right)}{\rho_{0}\left(\left(\Omega-c_{r}\right)^{2}+c_{i}^{2}\right)}\right\} \frac{|X|^{2} d r}{c_{i}\left(1+\frac{\left(\Omega-c_{r}\right)^{2}}{c_{i}^{2}}\right)} \\
= & \lim _{\epsilon \rightarrow 0} \lim _{c_{i} \rightarrow 0^{+}} \frac{m e^{2 m c_{i} t}}{2} \int_{r_{c}-\epsilon}^{r_{c}+\epsilon}\left\{\frac{D\left(\rho_{0} Z\right)}{\rho_{0}}-\frac{2\left(D \rho_{0}\right) \Omega^{2}\left(\Omega-c_{r}\right)}{\rho_{0}\left(\left(\Omega-c_{r}\right)^{2}+c_{i}^{2}\right)}\right\} \\
& \times \frac{\left(\tan ^{-1}\left(\frac{\Omega-c_{r}}{c_{i}}\right)\right)|X|^{2}}{(D \Omega)} d r \\
= & \lim _{\epsilon \rightarrow 0} \lim _{c_{i} \rightarrow 0^{+}} \frac{m e^{2 m c_{i} t}}{2}\left[\frac{D\left(\rho_{0} Z\right)}{\rho_{0} D \Omega}-\frac{2\left(D \rho_{0}\right) \Omega^{2}}{\rho_{0}(D \Omega)^{2}\left(1+\frac{c_{i}^{2}}{\left(\Omega-c_{r}\right)^{2}}\right)}\right]_{r_{c}} \\
& \times \int_{r_{c}-\epsilon}^{r_{c}+\epsilon} d\left(\operatorname { t a n } ^ { - 1 } \left(\frac{\Omega-c_{r}}{\left.\left.c_{i}\right)\right) d r}\right.\right.
\end{aligned}
$$

(by the use of the first mean value theorem for Riemann stieltjes integral).

This gives the following result.

Theorem 3.5. For non-monotonic angular velocity profiles the jump in the Reynolds stress across a critical layer is given by

$$
\left[r^{2} \tau\right]_{r_{c}}=\frac{m \pi}{2}\left\{\left[\frac{D\left(\rho_{0} Z\right)}{\rho_{0}}-\frac{2\left(D \rho_{0}\right) \Omega^{2}}{\rho_{0}(D \Omega)}\right]|X|^{2}\right\}_{r_{c}} \frac{1}{(D \Omega)_{c} \operatorname{sgn}(D \Omega)_{c}},
$$

where

$$
\operatorname{sgn}(D \Omega)_{c}= \begin{cases}+1 & \text { if }(D \Omega)_{c}>0 \\ -1 & \text { if }(D \Omega)_{c}<0\end{cases}
$$

\section{Concluding remarks}

The stability of swirling flows of inviscid, incompressible fluids with radius dependent density was initiated in Fung \& Kurzweg (1975). In Fung (1983) the Richardson number for swirling flows with velocity $(0, V(r), 0)$ and density $\rho_{0}(r)$ was defined and general analytical result, namely, a necessary condition for instability is that the Richardson number should be less than one quarter at least once was derived. This result is the swirling flow equivalent of the Miles-Howard theorem of the parallel flow theory. Moreover the semicircular and semielliptical instability theorems of the parallel flow theory were also extended in Fung (1983). It was noticed that in contrast to the parallel flow results these instability results are valid only for flows satisfying the condition $a b D \rho_{0} \geq 0$. Recently this problem has attracted the attention of some researchers because of the importance of this problem in the evolution of aircraft trailing vortices (see, for example, Dixit \& Govindarajan (2011) and Di Pierro \& Abid (2012)). These works on 
this problem focus on obtaining numerical and asymptotic results. Our interest in the present paper is to obtain general analytical results on this problem. In particular we have extended some results of Miles (1961) to the swirling flow context. We have found series solutions of the Fung and Kurszweg equation. The role of the Reynolds stress in transferring energy from the basic flow to the azimuthal disturbance is identified and the variation of the Reynolds stress for neutral modes is studied. It is found that the this variation is similar to the homogeneous swirling flow result of Maslowe \& Nigam (2008) whereby it is seen that the Reynolds stress varies like the inverse square of the radial distance than being a constant as in the parallel flow case. Finally it is proved in our paper that singular neutral modes do not exist when the Richardson number at the critical layer is greater than one quarter.

For density stratified parallel flows a viscous critical layer analysis has been developed recently in Maslowe \& Spiteri (2013) and the important role played by the Reynolds stress in the transfer of energy from a neutral mode to the basic shear flow is identified. They have also considered the critical layer analysis of swirling flows. However their swirling flow study is restricted to homogeneous flows only, but the disturbances considered are general three dimensional ones and not just the azimuthal ones. Still they have defined a Richardson number in this context which depends on the axial wave number. It is found that the role of the critical layer singularities of the linear inviscid stability equation and their effect on the variation of the Reynolds stress are significant in the computation of the neutral eigen functions. Moreover it is observed that the variation of the Reynolds stress would modify the azimuthal velocity profile and thereby change its stability properties.

For variable-density swirling flows a nonlinear critical analysis has been developed in Dattu \& Subbiah (2014b). It is found that the thickness of the nonlinear critical layer depends on the amplitude of the perturbation and the Reynolds number. The density variation of the basic flow enters the analysis through $J_{c}$, the value of the Richardson number at the critical layer. The main result of the nonlinear critical layer analysis is that the wave-induced vorticity inside the cell around the critical is a nonzero constant, in contrast to the homogeneous case where it is zero. It should be remarked here that the series solutions found in the present paper play a major role in the nonlinear critical layer analysis of swirling flows with variable-density.

\section{Acknowledgement}

The first author's work was supported by a UGC-SAP Fellowship which is duly acknowledged. We are thankful to the reviewers for their comments that helped us to substantially improve the presentation of our paper.

\section{References}

Chandrasekhar S 1961 Hydrodynamic and hydromagnetic stability. Oxford, UK: Oxford University Press Chossat P and Iooss G 1994 The Couette-Taylor problem, Spinger

Dattu H and Subbiah M 2014a Note on the stability of swirling flows with radius-dependent density with respect to infinitesimal azimuthal disturbances. ANZIAM J. 56(3): 209-232, 2015

Dattu H and Subbiah M 2014b On nonlinear critical layer analysis of incompressible swirling flows with radius-dependent density, J. Analysis (submitted)

Di Pierro B D and Abid M 2010 Instabilities of variable-density swirling flows. Phys. Rev. E 82(4): 046312

Di Pierro B and Abid M 2012 Rayleigh-Taylor instability in variable density swirling flows. Eur. Phys. J. $B$ 85(2): 1-8 
Dixit H N and Govindarajan R 2011 Stability of vortex in radial density stratification: Role of wave interactions. J. Fluid. Mech. 679: 582-615

Drazin P G and Reid W H 1981 Hydrodynamic stability. Cambridge: Cambridge University Press

Fung Y T 1983 Non-axisymmetric instability of a rotating layer of fluid. J. Fluid. Mech. 127: 83-90

Fung Y T and Kurzweg U H 1975 Stability of swirling flows with radius-dependent density. J. Fluid. Mech. 72: $243-255$

Howard L N 1961 Note on a paper of John W. Miles. J. Fluid. Mech. 10: 509-512

Howard L N and Gupta A S 1962 On the hydrodynamic and hydromagnetic stability of swirling flows. $J$. Fluid Mech. 14(03): 463-476

Kelly R E and Maslowe S A 1970 Nonlinear critical layer in a slightly stratified shear flow. Studies in Applied Mathematics 49(4): 301-326

Kochar G T and Jain R K 1979 Note on Howard's semicircle theorem. J. Fluid. Mech. 91(3): 489-491

Le Dizès S 2000 Non-axisymmetric vortices in two-dimensional flows. J. Fluid Mech. 406: 175-198

Maslowe S A and Nigam N 2008 The nonlinear critical layer for Kelvin modes on a vortex with a continuous velocity profile. SIAM J. Appl. Math. 68(3): 825-843

Maslowe S A and Spiteri R J 2013 A study of singular modes associated with over-reflection and related phenomena. J. Fluid Mech. 728: 120-145

Miles J W 1961 On the stability of heterogeneous shear flows. J. Fluid Mech. 10(4): 496-508

Spalart P R 1998 Airplane trailing vortices. Ann. Rev. Fluid Mech. 30(1): 107-138 